\title{
A New Vectorization Technique for Expression Templates in $\mathbf{C + +}$
}

\author{
J. Progsch and Y. Ineichen \\ Department of Computational Science \\ ETH-Zürich \\ CAB H 83.2 \\ Universitätstrasse 6 \\ 8092 Zürich SWITZERLAND
}

\author{
A. Adelmann \\ Paul Scherrer Institute \\ 5232 Villigen PSI \\ SWITZERLAND
}

\begin{abstract}
Vector operations play an important role in high performance computing and are typically provided by highly optimized libraries that implement the Basic Linear Algebra Subprograms (BLAS) interface. In $\mathrm{C}++$ templates and operator overloading allow the implementation of these vector operations as expression templates which construct custom loops at compile time and providing a more abstract interface. Unfortunately existing expression template libraries lack the performance of fast BLAS implementations. This paper presents a new approach - Statically Accelerated Loop Templates (SALT) - to close this performance gap by combining expression templates with an aggressive loop unrolling technique. Benchmarks were conducted using the Intel C++ compiler and GNU Compiler Collection to assess the performance of our library relative to Intel's Math Kernel Library as well as the Eigen template library. The results show that the approach is able to provide optimization comparable to the fastest available BLAS implementations, while retaining the convenience and flexibility of a template library.
\end{abstract}

\section{INTRODUCTION}

Vector and matrix operations are important building blocks of numerical computations like solving a system of linear equations or integrating differential equations. Providing optimized implementations of these operations is therefore of great interest. Naive implementations are usually oblivious to hardware features and limitations that affect the performance and are subject to compiler optimization which may vary greatly across different compilers and optimization settings. The Basic Linear Algebra Subprograms (BLAS) interface with roots in the FORTRAN programming language has become the quasi standard for libraries that provide such optimized linear algebra routines. Examples for such libraries are the Automatically Tuned Linear Algebra
Software (ATLAS), GOTO BLAS or Intel's Math Kernel Libraries (MKL) [1]. In the last decade FORTRAN has been overtaken by $\mathrm{C} / \mathrm{C}_{+}+$as the primary language for scientific software and accordingly $\mathrm{C} / \mathrm{C}++$ bindings for BLAS libraries are being used to benefit from the optimized implementations. Unfortunately this approach falls short when language inherent features of $\mathrm{C}++$ like operator overloading or templates are being used. More modern approaches are present in expression template libraries such as Blitz++ [2], Eigen [3] and the Portable Expression Template Engine (PETE) [4].

One added benefit of the expression template approach is the compile time construction of optimized loops. These allow, e.g., a reduction of memory accesses of operations that have to be expressed as multiple calls to BLAS routines but could be 


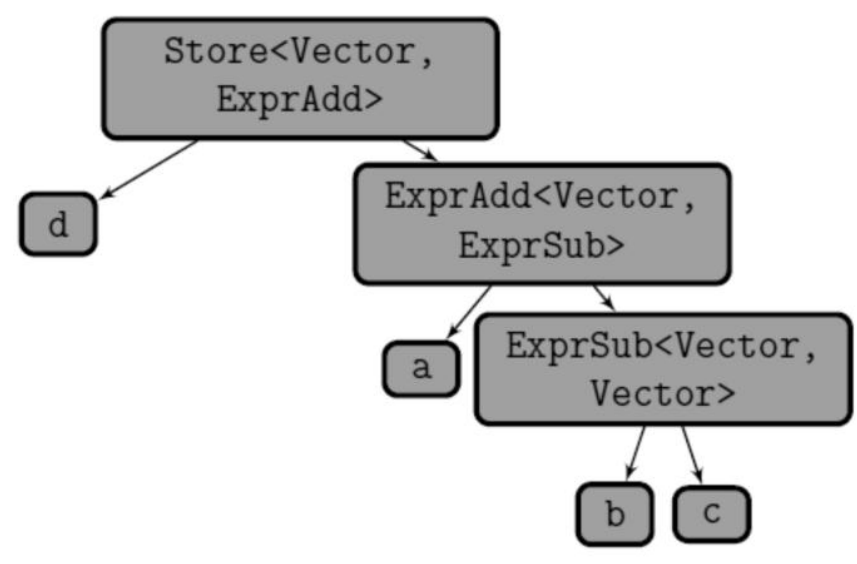

Figure 1. Expression tree for $d=a+(b-c)$.

written as a single loop, which is exactly what the expression template library does (loop fusion). Generic expression template implementations still rely on the compiler to apply additional optimization like the use of Single Instruction Multiple Data (SIMD) instructions (vectorization) or loop unrolling. Explicit vectorization for expression templates can be achieved with intrinsics which allow the emission of specific CPU instructions without the use of inline assembler. Apart from using the right instructions, the key to close to optimal performance is aggressive loop unrolling and instruction ordering that minimizes pipeline stalling. Loop unrolling can be achieved by template meta programming which tends to produce suboptimal register usage while the loop unrolling capabilities of the compilers are inconsistent and still not optimal $[5,6]$.

In sections II and III we will discuss expression templates and vectorization. Section IV introduces Statically Accelerated Loop Templates (SALT) [7] and explains the ideas behind our optimization technique and presents some implementation details. In sections $\mathrm{V} \& \mathrm{VI}$ we discuss measurements and an outlook of further possible parallelization strategies by means of OpenMP and Message Passing Interface (MPI). Our final conclusions are presented in section VII.

\section{EXPRESSION TEMPLATES}

Expression templates $[5,6,8]$ were invented independently by Todd Veldhuizen
$[9,10]$ and David Vandevoorde. This implementation technique uses the static evaluation abilities of modern $\mathrm{C}++$ compilers together with templates to construct a static expression tree at compile time as shown in Figure 1. This is typically used for loop fusion. Instead of having every part of the expression individually loop over the data and perform an operation, only one single loop is produced that performs the composite expression. This helps reduce the loop overhead (increments and conditional jumps), can reduce the amount memory accesses by keeping intermediate results in registers and removes the need to allocated temporary objects. Through the use of template meta programming it is even possible to manipulate the expression tree at compile time, e.g., to apply algebraic transformations. While the nodes of the expression tree are technically template classes their entirely static nature allows the compiler to inline everything. In consequence the creation of instances of these classes does not incur any overhead in the resulting machine code. This implies that one can think of expression templates as a form of code generation at compile time.

The downsides of expression templates are that they increase compile times, the binary size and tend to produce hard to read error messages. Compile times and binary sizes are usually of no great concern given availability of memory and the performance of modern compilers. The error messages $\mathrm{C}++$ compilers produce in connection with intricate template constructs 
on the other hand are a serious handicap. Seemingly trivial syntax errors can result in a cascade of hard to decipher errors and warnings.

Expression templates are implemented by returning abstracted operation objects in operators and functions that contain all necessary information, instead of calculating the result themselves. For example the addition operator will return an AddExpr object that has references to the operands and an evaluation function. The operands in turn can also be expression objects. Whit this approach complex expressions are turned into an expression tree at compile time. Accordingly we will refer to expression objects as nodes in an expression tree. The expression tree is only evaluated when the actual need arises (lazy evaluation). Typically this happens in the assignment operator or in functions like the dot product or norm. The actual loop is contained in these functions and evaluates the expression tree for every element it needs. This also means that results that are not requested are never computed.

\section{VECTORIZATION}

Most modern general purpose processors over a set of vector instructions that operate on registers that contain multiple operands which allow instruction level parallelism Single Instruction Multiple Data (SIMD). These instructions are instrumental for the optimization of vector operations since the attainable performance is usually a multiple of what is offered by the more conservative Floating Point Unit (FPU) instructions. To profit from SIMD instructions in $\mathrm{C}++$ one can either rely on the compiler to detect loops that can be vectorized or enforce SIMD instructions with inline assembler or intrinsics rendering the code non-portable. Since inline assembler does not have a uniform syntax across compilers it is not well suited for use inside a template library. Intrinsics for Intel's Streaming SIMD Extension (SSE) instruction set on the other hand integrate well into standard $\mathrm{C}_{++}$and are supported by most compilers. Our template library wraps the intrinsics and the vector data types into a vectorizer template class, hence the actual implementations of the algorithms are independent of the underlying instruction set. They only require a specialized instance of the vectorizer template for each targeted platform. In the present form the library only contains a specialization for Intel's SSE instruction set.

\section{IMPLEMENTATION}

\section{a. General Idea}

The study of existing vectorization methods in numeric libraries and simple experiments showed that the main performance gain of hand optimized BLAS implementations comes from heavily unrolled loops and instruction reordering. While it was relatively easy to have a compiler emit the "optimal" instructions via intrinsics we lacked the ability to unroll the loops in a controllable fashion. Even if the compiler did unroll the loops there was no way to consistently control the relative order or multiple sets of memory and operation instructions.

The main idea to achieve the required control over the loop structure was to use abstracted loop templates. These contain calls to functions like load, store and operation which are provided by the expression tree. This approach separates the instructions that are executed from the order in which they are executed and gives the control over both aspects to the library implementer in place of to the compiler.

The SALT has three main components which are used to assemble the final loop (see Figure 2). The vectorizer class provides the platform specific vector instructions, the loop templates provide the loop structure and the expression tree defines the operation that is to be performed. These three components are then used to assemble the actual loop inside an execute function that takes the expression tree as its sole argument and chooses an appropriate loop template while the vectorizer is implicitly chosen by the type parameter of the participating vectors.

\section{b. Instruction Decomposition and Local Storage}

The expression tree nodes have to provide all the necessary methods and variables that are required for the loop. The operations that are used inside the loops are separated into load, store and 


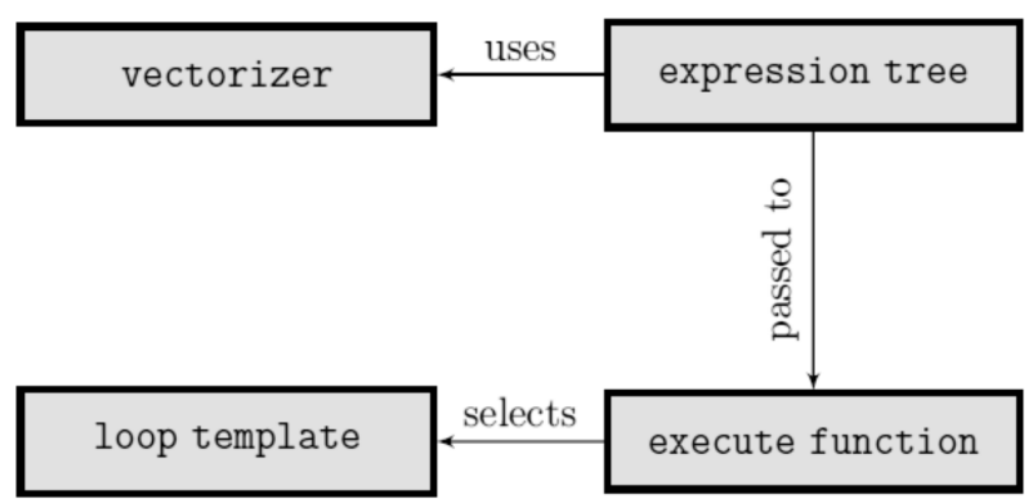

Figure 2. Component interaction.

operation. The operation functions are further subdivided into vector op and single_op. single_op is used to finish up operations where the amount of data is not a multiple of the SIMD vector size. Additionally there are the init and load once functions that are called once at the beginning of the loop to initialize local variables and possibly perform calculations that have to be performed only once for the whole loop. The difference between init and load_once is that init is called exactly once for the whole loop while load once is called every time the loop is unrolled. The cleanup function complements init. Some expression nodes contain an additional reduction function which is used to reduce multiple local variables to a single result. In addition to the functions defining the operation to be performed the loop may require local variables for intermediate results. When the loop is unrolled it might even require one set of variables for each time it was unrolled. Additionally there can be variables that are only needed once for the whole loop independent of the unrolling. Since the loop template cannot declare an arbitrary number of local variables it instead declares a fixed amount of variables (one for every time the loop is unrolled) with a composite type Storage that is also provided by the expression tree. Similarly there is a type TemporaryStorage that is only instantiated once by the loop template. Listing 1 shows the basic interface of an expression node and Listing 2 shows an example loop template that is unrolled twice.

\section{c. Loop Structure}

To attain near optimal performance the loop has to be structured to minimize pipeline stalls. This can be achieved by using as many registers as possible and by interleaving instructions such that the distances between the usage of each individual register is maximized. This is done by grouping instructions into packages and starting each instruction package with a burst of load commands followed by a burst of operation commands and finishing with a burst of store commands, while retaining the relative order of variable/register usage inside each burst. By the time the operation command is called on the first register the load command has had the maximum possible amount of time to complete. Also grouping load and store instructions helps the CPU to optimize memory bus usage.

To ensure optimal register usage the instruction package size has to be chosen depending on the amount of registers used by each operation. This number can be retrieved from the expression tree and the appropriate loop template can be chosen at compile time. For that purpose the run function as seen in Listing 2 is a member function of a template struct that is specialized for different register usage patterns.

A single iteration of the unrolled loop can contain multiple instruction packages. The number of packages per loop was determined by trial. Choosing low numbers of packages (usually only one) gives high 


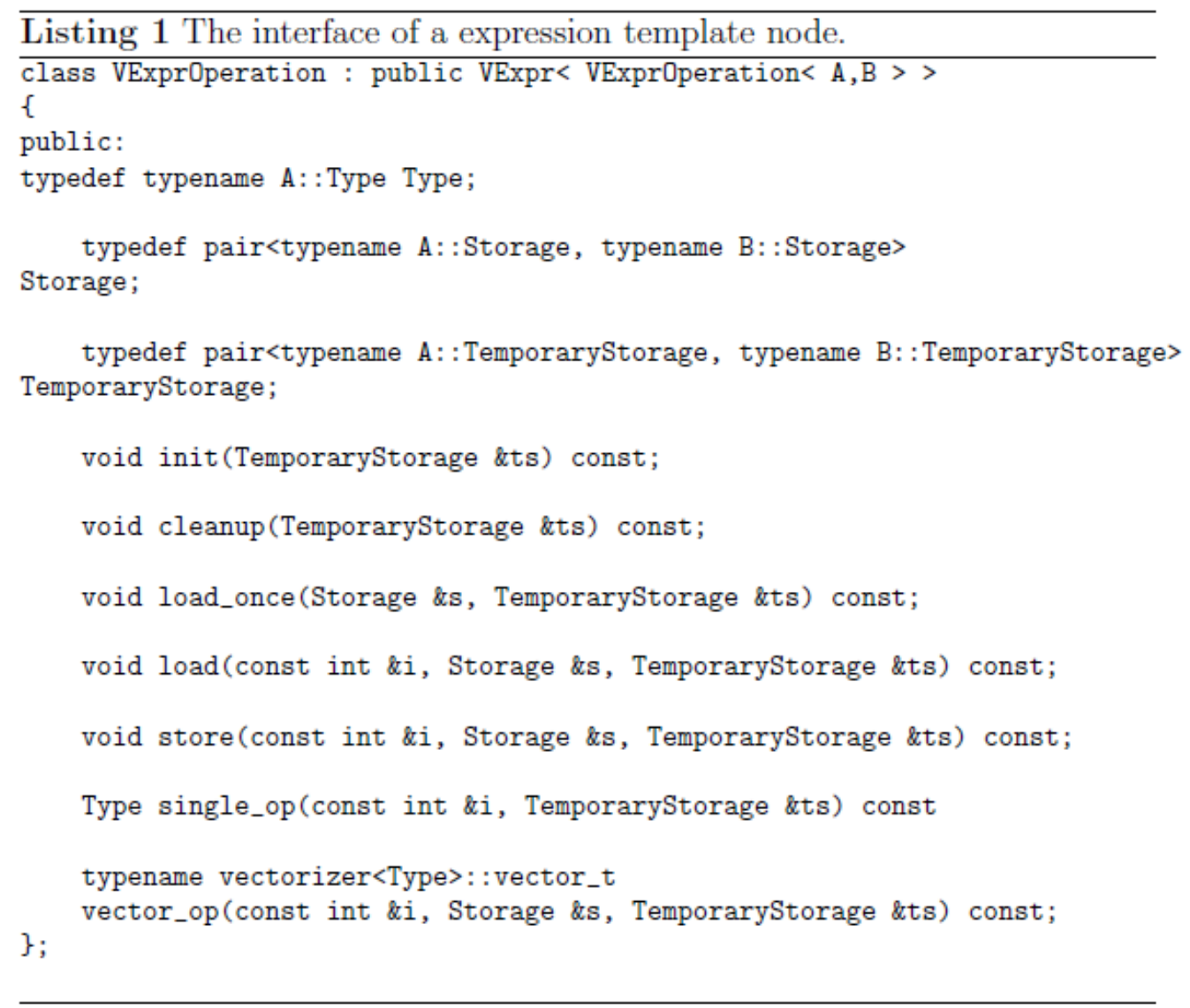

performance for small vector sizes while higher numbers yield better performance for large vectors. We chose relatively high numbers of packages for SALT since those approximate the performance of BLAS implementations better.

\section{PERFORMANCE BENCHMARKS}

Measurements are conducted for three common vector operations: the dot product (sDOT), vector scaling (SSCAL) and operations of the form $y=y+\alpha x$ (sAXPY). Tests were conducted by repeatedly executing the operation on the same two (or in case of SSCAL one) vectors. For the purpose of checking the correctness of the results, the elements of the input vectors were set equal to their index. The results were compared to Intel's MKL library (BLAS interface) and to Eigen (expression templates). MKL was chosen for being one of the best performing BLAS implementations for the Intel platform. Eigen was chosen for being one of the most established template libraries and for using a very similar approach of optimization with intrinsics. The results for a Intel Core i5580M CPU (2.66 GHz, $3.33 \mathrm{GHz}$ TurboBoost) are shown in Figures 3, 4 and 5. Additionally Figure 6 shows the results for an out-of-place vector scaling operation $(y=\alpha x)$ which cannot be expressed as a single BLAS call and therefore has to be written as combination of a copy and a scaling operation when using BLAS but gets compiled into a single loop by the expression templates. Compiler version and optimization flags for both compilers are shown in table 1 . The version of SALT that was used for the benchmarks is available at [7].

\section{a. Discussion}

The graphs show, that SALT is capable of matching and even exceeding the performance of $\mathrm{MKL}$ and is able to 


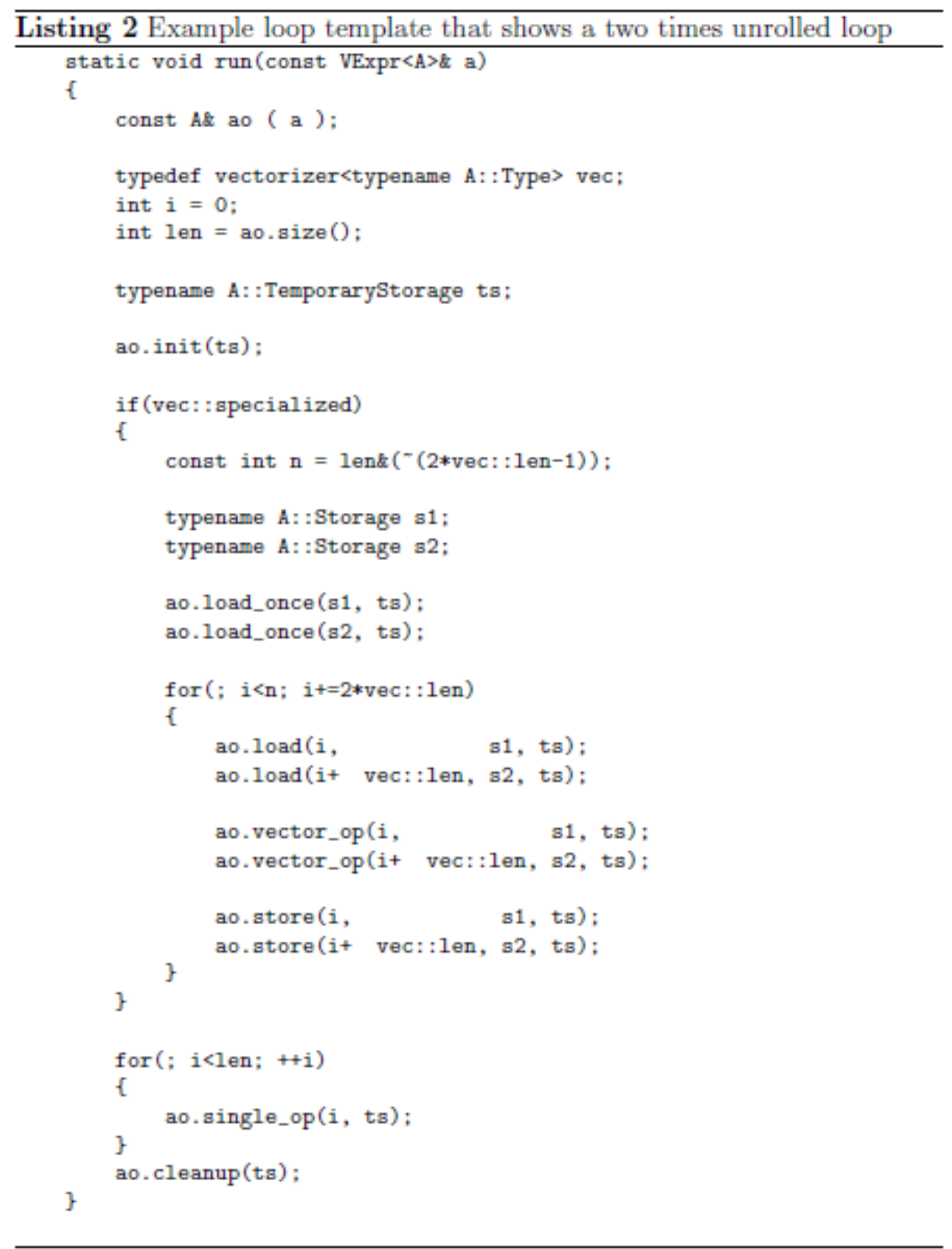

deliver significantly higher peak performances than Eigen. For small vectors Eigen is capable of delivering higher performance than both SALT and MKL which is likely caused by lower overhead since the loop unrolling requires more one time branch instructions, especially in cases where the vector is shorter than the length of a single loop iteration. Also MKL, being a precompiled library, cannot profit from aggressive inlining and call optimizations to the same extent as the template libraries and has to cover more general cases like unaligned and strided data which further increases overhead. The results for the out- of-place scaling operation (Figure 6) demonstrates the ability of expression templates to reduce the amount of memory accesses that are caused by operations that require multiple BLAS calls. Since these vector operations are mostly bound by memory bandwidth, SALT outperforms MKL in this case by up to factor two.

\section{HIGHER LEVEL PARRALIZATION \\ The usage of SIMD operations already provides instruction level parallelism, but one might be interested in additional parallelization on the thread and}




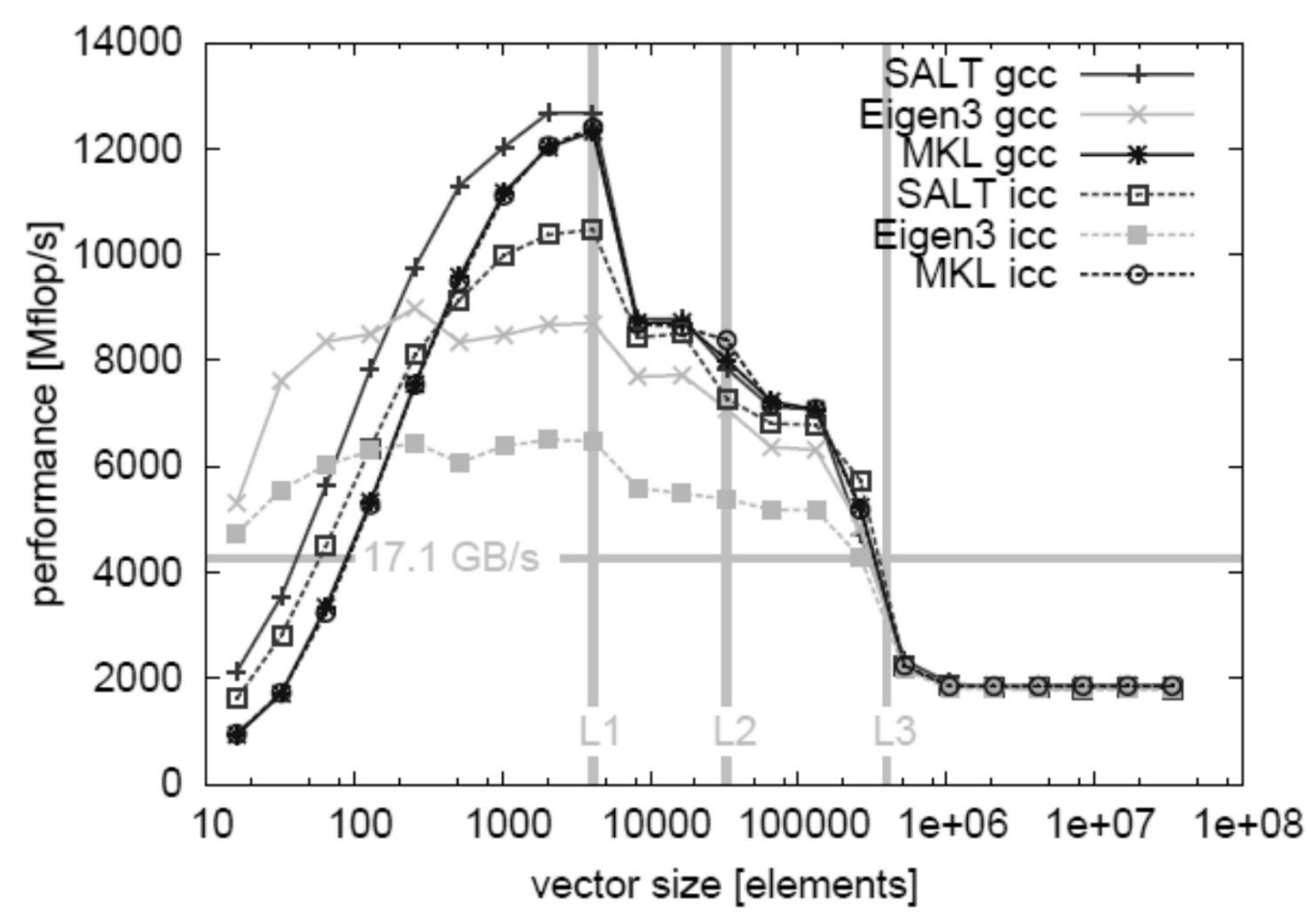

Figure 3. Performance results for the single precision dot product (sDOT) on an Intel Core i5$580 \mathrm{M}$ CPU. The gray lines indicate the sizes of the different caches and the $17.1 \mathrm{~GB} / \mathrm{s}$ memory bandwidth of the processor used.

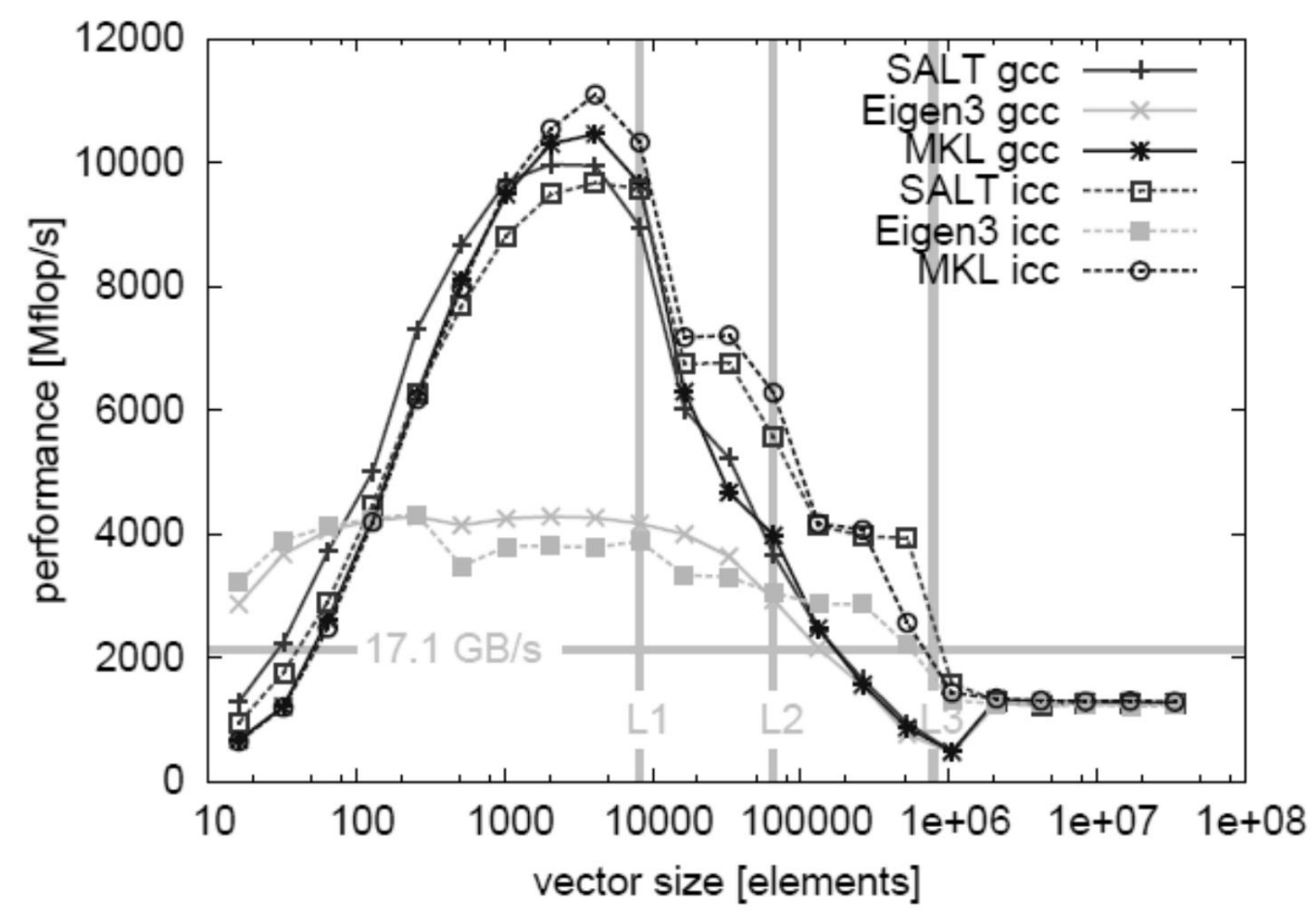

Figure 4. Performance results for the single precision vector scaling operation (SSCAL) on an Intel Core i5-580M CPU. The gray lines indicate the sizes of the difference caches and the 17.1 $\mathrm{GB} / \mathrm{s}$ memory bandwidth of the processor used. 


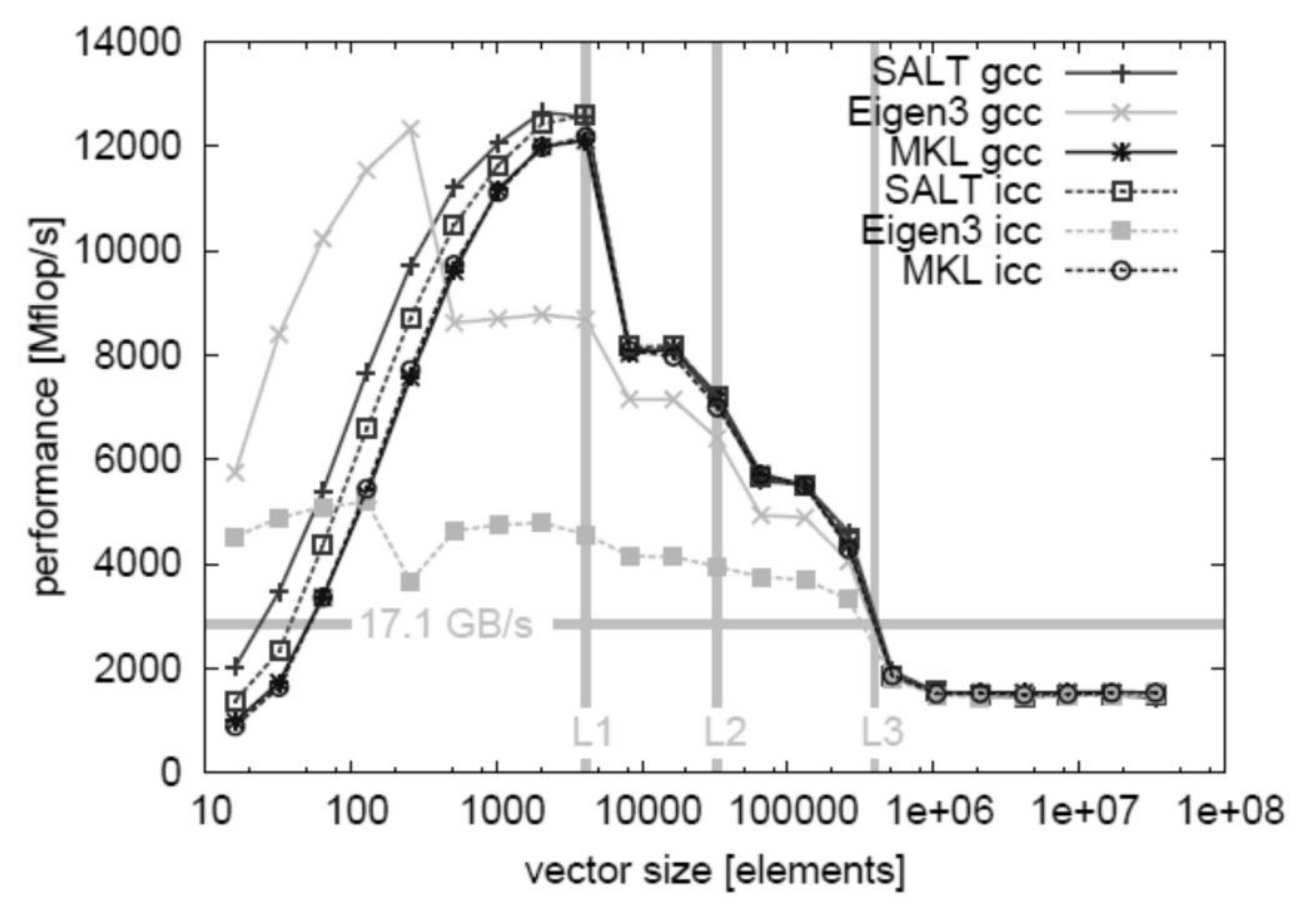

Figure 5. Performance results for the single precision operation $y=y+\alpha x$ (sAXPY) on an Intel i5-580M CPU. The gray lines indicate the sizes of the different caches and the $17.1 \mathrm{~GB} / \mathrm{s}$ memory bandwidth of the processor used.

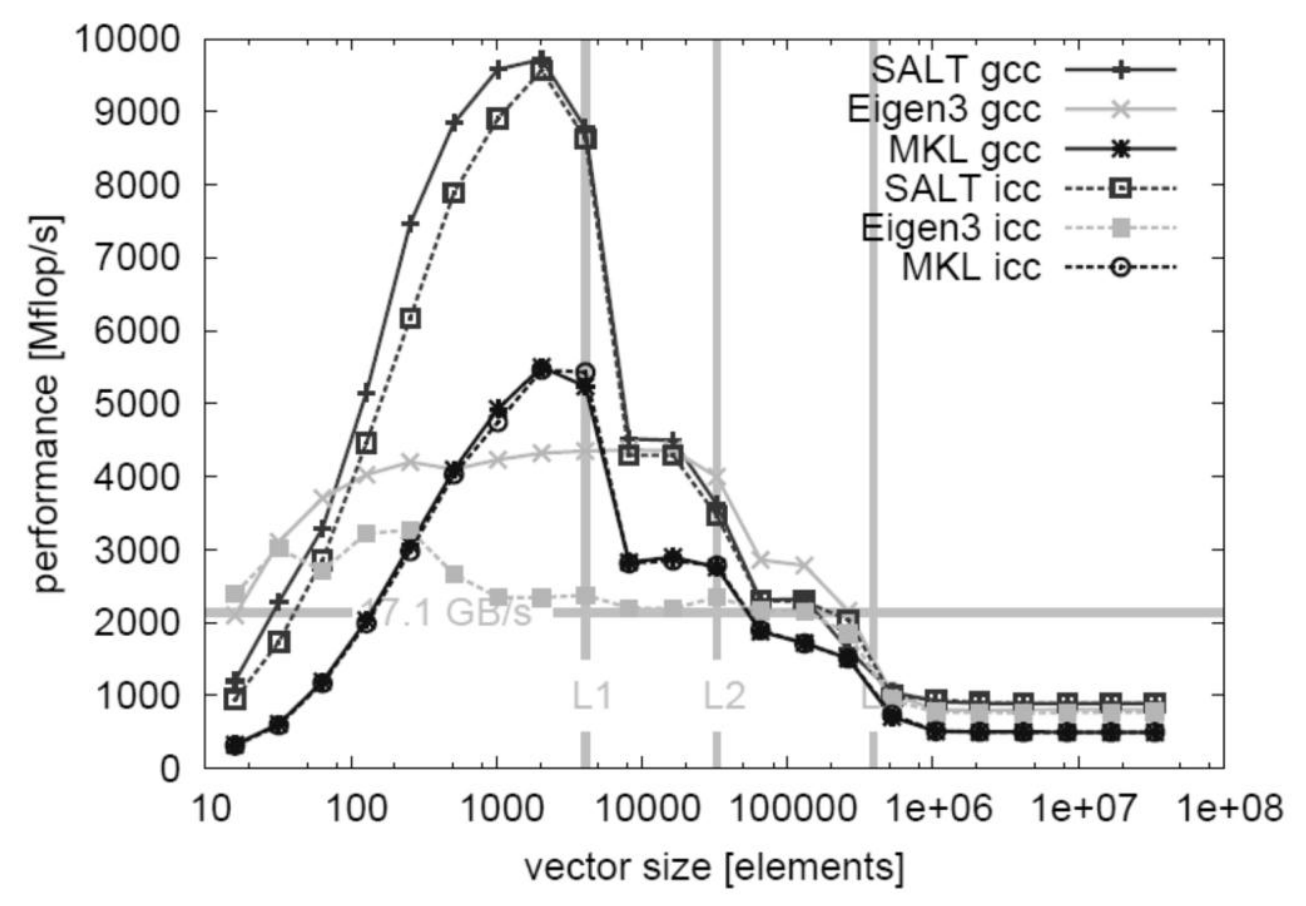

Figure 6. Performance results for the single precision out-of-place vector scaling operation (sCOPY + sSCAL) on an Intel Core i5-580M CPU. The gray lines indicate the sizes of the different caches and the $17.1 \mathrm{~GB} / \mathrm{s}$ memory bandwidth of the processor used. 


\begin{tabular}{|c|c|c|}
\hline Compiler & Version & Flags \\
\hline GCC & 4.5 .2 & $-03-$ msse3 \\
ICC & 12.0 .2 & $-03-$ msse3 \\
\hline
\end{tabular}

Table 1. Compiler versions and flags.

process level. Thread level parallelization, which is attractive for modern multicore architectures, could be achieved easily by inserting OpenMP pragmas into the loop templates. When spawning additional threads one has to be careful to avoid false sharing and excessive overhead. For small sized vectors that $t$ into the L1 cache of the processor the execution times of the total operation is in the order of a few hundred thousand cycles. Spawning a thread each time the operation is executed will often have the opposite effect of reducing performance by introducing overhead. In the case of very large vectors it has to be noted that the rate at which even a single core is able to process data usually exceeds the memory bandwidth of the system. The sDOT (Figure 3) example demonstrates this by achieving a peak throughput of about 60 $\mathrm{GB} / \mathrm{s}$ for vectors that completely fit into the $\mathrm{L} 1$ cache while the maximum memory bandwidth of the used processor amounts to only 17.1 GB/s. In cases where the operation is already limited by memory bandwidth on a single core, using multiple cores that share a memory bus will not increase performance. Better results might be achieved by parallelizing "outside" of the vector library.

Process level parallelization using a data parallel ansatz and MPI communication-which is popular in software for cluster computers-can also be provided by an expression template library. Since the data parallel ansatz does not directly affect how the individual operations are carried out in each process, it is best handled by an additional abstraction layer thus enforcing the single responsibility principle.

\section{CONCLUSIONS}

The benchmarks show that our new approach-Statically Accelerated Loop Templates (SALT) -allows template libraries to match the performance of BLAS libraries and even outperform them in cases that require the composition of BLAS calls. Performance inconsistencies across different compilers are greatly reduced in comparison to existing template libraries. SALT retains the math-like syntax and better integration into standard $\mathrm{C}_{++}$that comes with using $\mathrm{C}++$ specific features like operator overloading and generic programming, and allows existing template algorithms to instantly benefit from efficient vectorization. The strong separation of low level instructions, expression building and instruction ordering into the vectorizer class, expression nodes and loop templates gives unique access points for each aspect of the algorithms and therefore simplifies customization and extension of the framework by following the single responsibility principle. Additionally the pure template character of the library makes it easy to use and lightweight since no additional libraries have to be linked or compiled.

\section{REFERENCES}

1. Intel. Intel math kernel library. http://software.intel.com/en-us/articles/ intel-mkl/.

2. Blitz++. http://www.oonumerics.org/ blitz/.

3. Gaël Guennebaud, Benoît Jacob, et al. Eigen v3. http://eigen.tuxfamily.org, 2010.

4. PETE. http://acts.nersc.gov/formertools/ pete/index.html.

5. C. Pflaum and Z. Rahimi. "Parallelization of Staggered Grid Codes with Expression Templates." Journal on Computational Science and Engineering (2009).

6. J. Härdtlein, C. Pflaum, A. Linke, and H.Wolters. "Advanced Expression Templates Programming." Computing and Visualization in Science (2009).

7. SALT source code http://amas.web.psi.ch/people/ aadelmann/pub/SALT.tar.gz.

8. J. Härdtlein. Moderne Expression Template Programmierung-Weiterentwickelte Techniken und deren Einsatz zur Lösung Partieller Differentialgleichungen. $\mathrm{PhD}$ thesis, Univ. Erlangen (2007).

9. Todd Veldhuizen. "Expression templates." C++ Report 7 (1995). 
10. Todd Veldhuizen. "Arrays in Blitz++." In: Proceedings of the 2nd International Conference on Scientific Computing in

Object-Oriented Parallel Environments (1998

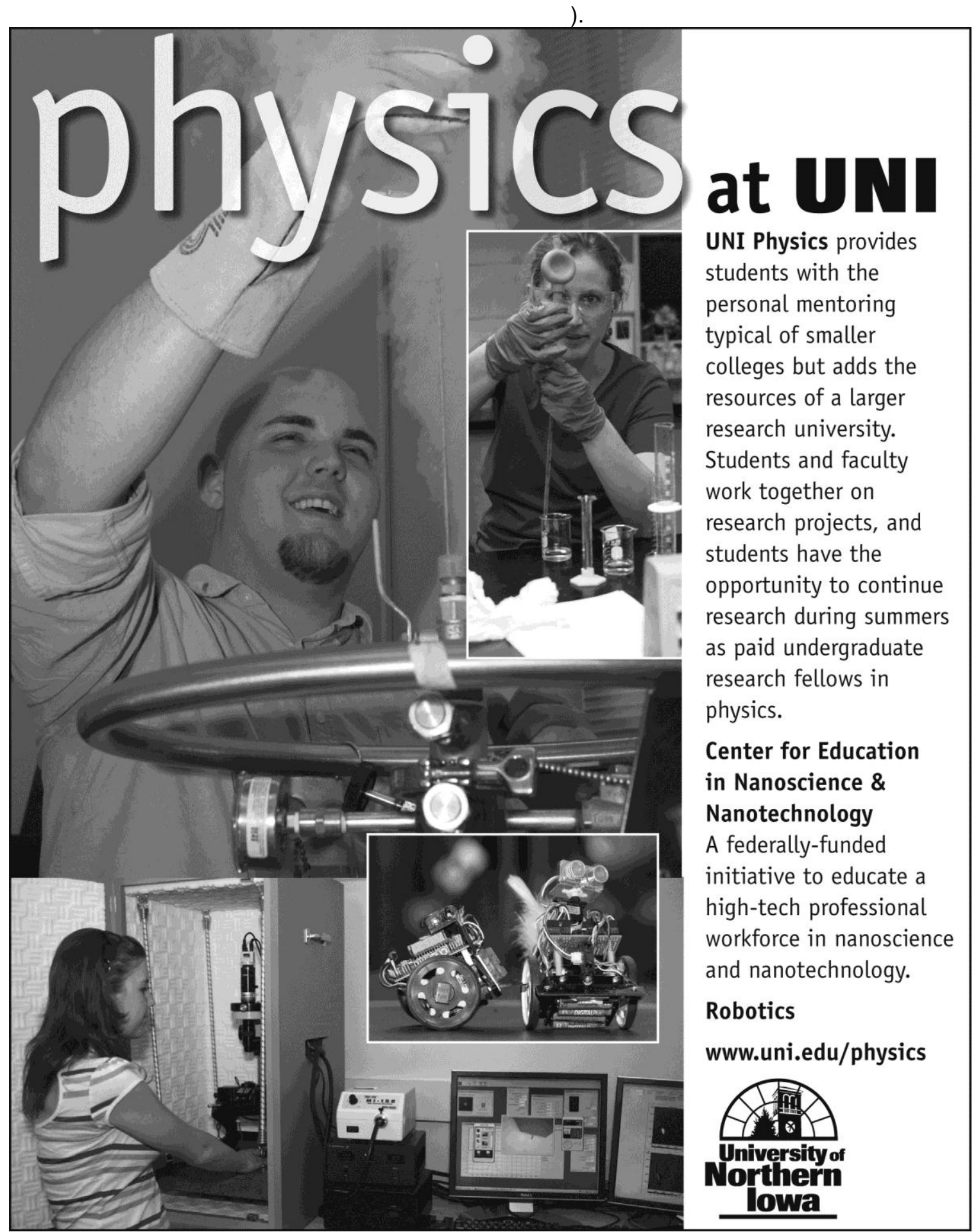

